Volume 9, No.5, September - October 2020

International Journal of Advanced Trends in Computer Science and Engineering

Available Online at http://www.warse.org/IJATCSE/static/pdf/file/ijatcse74952020.pdf

https://doi.org/10.30534/ijatcse/2020/74952020

\title{
Computer Vision and Machine Learning based Facial Expression Analysis
}

\author{
Balasundaram A ${ }^{1}$, Ashokkumar $\mathbf{S}^{2}$, Sasikumar A N ${ }^{3}$,Kajendran $K^{4}$,P.J.Sathish Kumar ${ }^{5}$ \\ ${ }^{1}$ Assistant Professor, School of Computer Science and Engineering, Center for Cyber Physical Systems, \\ Vellore Institute of Technology (VIT), Chennai, Tamil Nadu, India, balasundaram2682@gmail.com \\ ${ }^{2}$ Assistant Professor, Department of CSE, Saveetha School of Engineering, SIMATS, Chennai, Tamil Nadu, India, \\ sabariashok2016@gmail.com \\ ${ }^{3}$ Sasikumar A N, Associate professor, Department of CSE, Panimalar Engineering college, Chennai, Tamil Nadu, \\ India,ansasikumar@gmail.com \\ ${ }^{4}$ Kajendran K, Associate professor, Department of CSE, Panimalar Engineering college,Chennai, Tamil Nadu, \\ India, kajendran@yahoo.com \\ ${ }^{5}$ P.J.Sathish Kumar, Associate Professor, Department of CSE, Panimalar Engineering college,Chennai, Tamil \\ Nadu, India,sathishjraman@gmail.com
}

\begin{abstract}
Human facial expression plays a pivotal role in depicting the mindset of an individual. The facial expression clearly conveys the change in mood of a person. Facial expression is considered as one of the natural means to study human mind and psyche. Hence analyzing facial expression creates great interest among researchers working in the area of computer vision. This work is focused towards analyzing and automatically classifying the facial expression depicted by a human face in a given image. To accomplish computer vision and machine learning algorithms are deployed. The facial expressions are classified into seven different expressions and results are discussed in detail
\end{abstract}

Key words :Face Expression Analysis, Face Expression Classification, Face Expression Detection.

\section{INTRODUCTION}

There is a common saying which states that Face is the index of mind. Face conveys the instantaneous mood of the person through the expressions. In the current era, our dreams have gone high and it binds no bounds. Larger studies and work is carried out in the field of digital picture and image processing which forms the basis of Computer Vision (CV). The manner of development has been exponential and it's ever increasing. Image Processing is an enormous place of studies in the cutting-edge world and its packages are very widespread.

Image processing is the field of signal processing where both the input information and output alerts are images. One of the most vital applications of Image processing is Facial expression popularity. Our emotion is discovered by the expressions in our face. Facial Expressions plays an important function in interpersonal communication. Facial expression is a non-verbal clinical gesture which gets expressed in our face as in step with our feelings. Automatic popularity of facial features plays a crucial role in artificial intelligence and robotics and as a consequence, it is a need of the generation. The expressions are expressed in the form of facial actions such as laughter, cry, sigh, grin etc. These actions are sometimes called as facial gestures. Figure 1 lists down some of the common facial gestures.

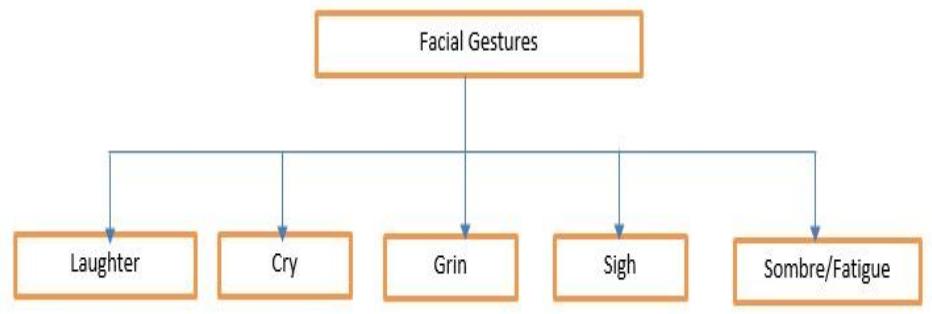

Figure 1: Some Common Facial Gestures

These facial gestures are pivotal in determining the facial expressions. Typically, facial expressions can be classified into seven categories namely:

- Anger

- Disgust

- Worry (or) Sorrow (or) Sad

- Happiness (or) Joy

- Disappointment (or) Anguish

- $\quad$ Surprise (or) wonder

- Neutral

Figure 2 shows the different types of classification of facial expressions and Figure 3 shows some real-time visuals of these facial expressions. 


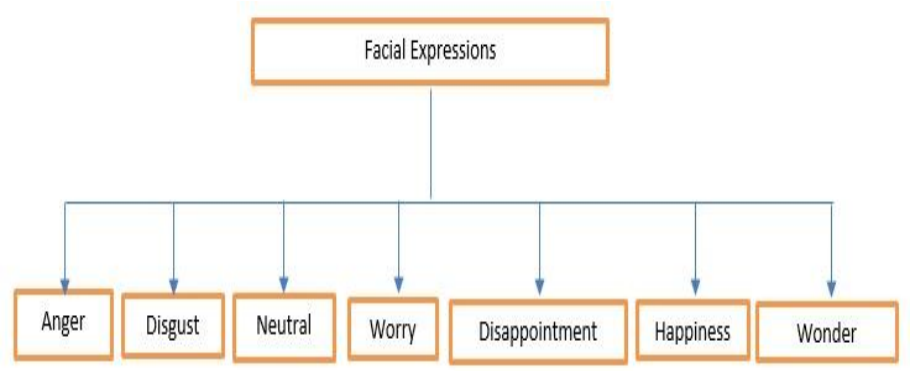

Figure 2: Classification of Facial Expressions

This work is focused towards extracting the facial features from a given image and classification of the same into the above discussed facial expressions using computer vision and machine learning.

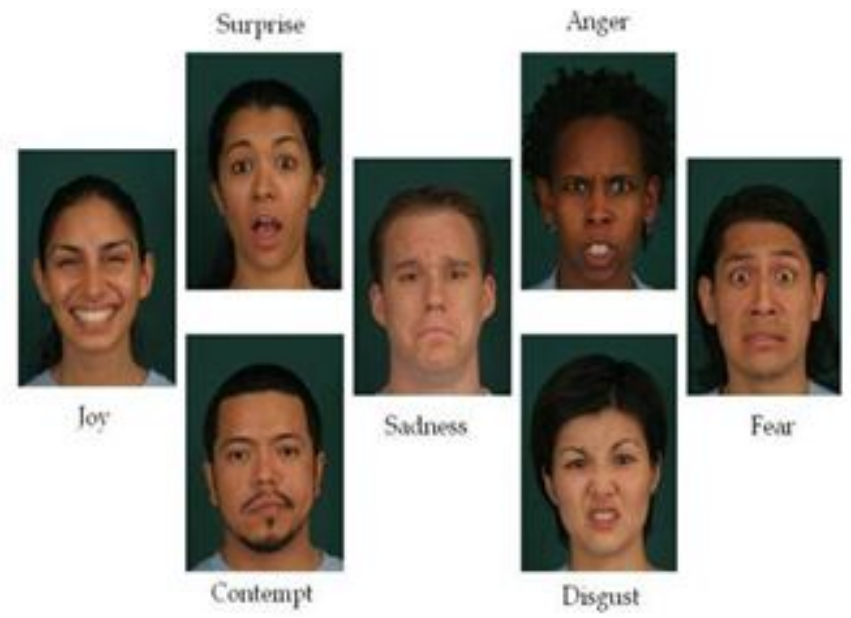

Figure 3: Real Time Facial Expressions

\section{RELATED WORKS}

Computer Vision is an emerging technology and a sub discipline of Artificial Intelligence (AI). Computer Vision deals with how computers can gain high level understanding from digital images and videos. This understanding is acquired through several analysis such as image analysis, video analysis and sequence analysis. Computer Vision is applied across many domains such as health care, education, video analytics, video surveillance etc., as illustrated in [13-18].

Machine Learning is a concept that allows the system to learn from examples and experience, and that too without being explicitly programmed. Most of the AI works use machine learning algorithms for classification. Some commonly used machine learning algorithms are shown in figure 4.

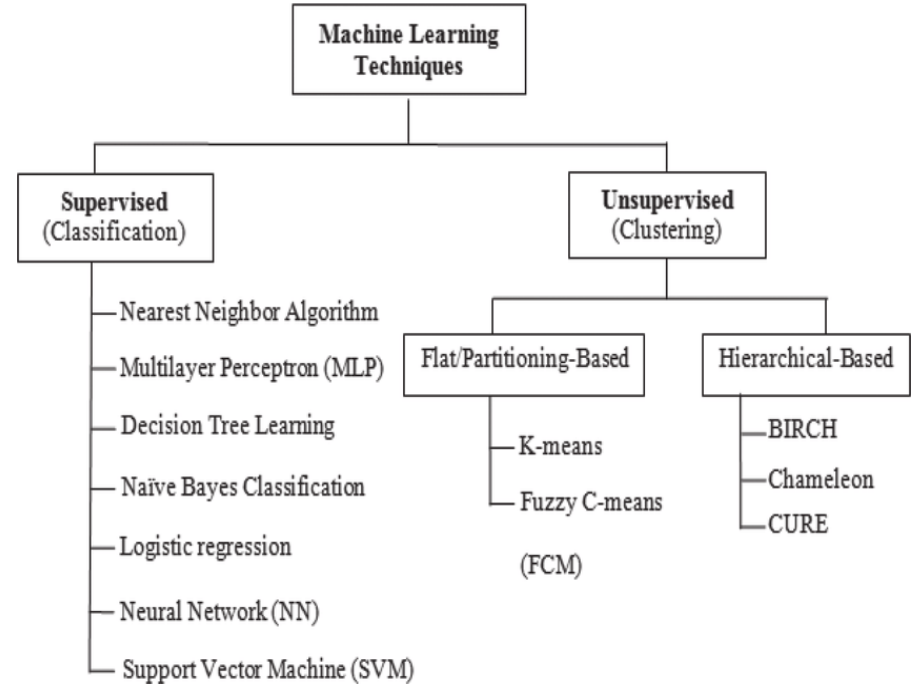

Figure 4: Machine Learning Algorithms Used for Classification

Several works which relate computer vision and cloud environment [13-17] have been carried out. This section lists some of the works carried out towards facial expression analysis in the recent years. M. Pantic et al. [1] have used facial muscle actions to analyze the different types of facial expression. Y. Li et al. [2] have explored the possibilities of simultaneous facial feature tracking and subsequently using these features to classify the facial expressions. M. Dahmane and J. Meunier [3] have developed a prototype model that uses SIFT feature to analyze facial expressions.

M. Song et al. [4] have used image ratio and intensity features to classify different facial expressions. M. Pantic and L. J. M. Rothkrantz [5] have used a multidetector approach to facial feature localization and facial expression classification in static images. I. Kotsia and I. Pitas [6] used a combination of Support Vector Machines (SVM) and Geometric deformation features to analyze facial expressions.

D. Li et al [7] have used generative adversarial network to perform multi-pose facial expression recognition. A computer vision and optical flow based facial motion model was developed by I. A. Essa and A. P. Pentland [8] to analyze facial expressions. Adena J. Zlochower [9] have used a computer vision based strategy to analyze parent-infant interaction. MingliangXue et al. [10] have suggested a granular description of facial expression intensity in which characterization of motion-based facial expression features is presented by a collection of information from features. A new metric learning method based on histogram data classification was proposed by Hamid Sadeghi et al. [11] to classify facial expressions. Feng Zhou et al. [12] used a dimensional emotion model to perform fine grained facial expression analysis.

\section{PROPOSED SYSTEM}

The proposed approach uses a combination of Computer Vision and Machine learning to accomplish the task of facial 
expression analysis. The flow of the proposed system is shown in figure 5. It comprises of the following stages:

- Image Pre-processing

- Face Detection

- Facial Feature Extraction

- Emotion Detection and Classification

- Output Expression

Image Preprocessing is the first step in the proposed approach. The primary objective of this pre-processing step is to reduce the noisy data and to enhance the image characteristics so that the facial components can be easily detected for further analysis. Figure 6 lists the basic steps involved in the pre-processing.

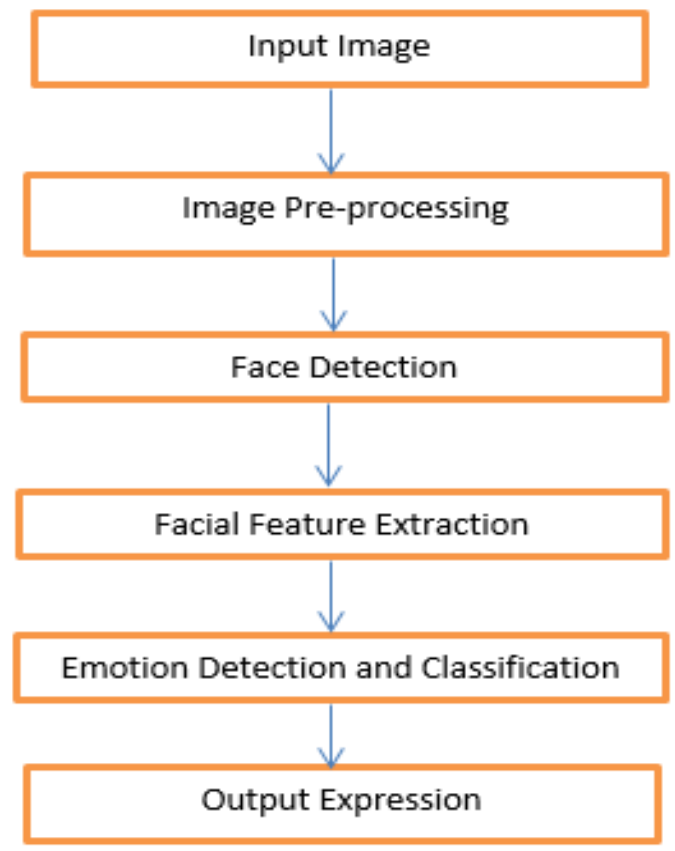

Figure 5: Steps Involved in the proposed system

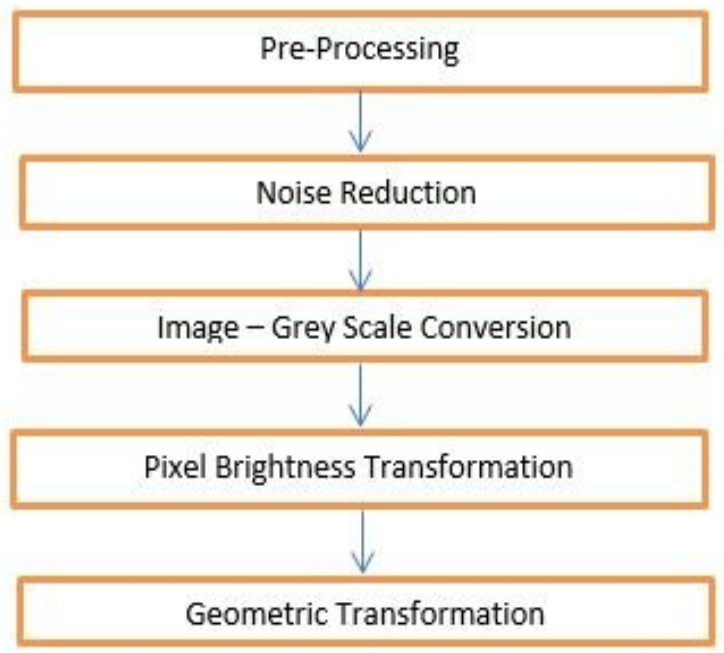

Figure 6: Steps in Image Pre-processing
The pre-processing step is followed by face detection and localization. This is followed by facial feature extraction which is a critical step in face recognition and is defined by the manner of locating specific areas, points, landmarks, or curves/contours in a given 2D picture or a $3 \mathrm{D}$ range image. Typical facial features that are extracted for face expression detection are shown in figure 7 . These facial features include nose, mouth, eyes etc.

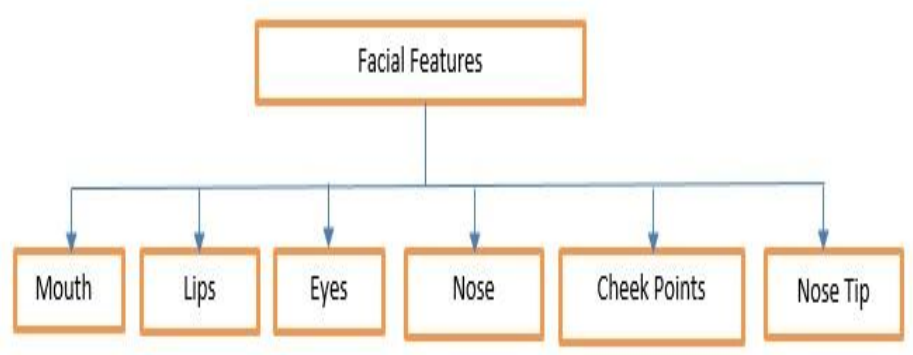

Figure 7: Typical Facial Features Extracted

The next significant step towards facial expression analysis is emotion detection and classification. Emotion is detected by analyzing the facial features and the dimensions of each feature such as size, shape, color etc. The emotion classification is performed using a Machine Learning classifier namely Support Vector Machines (SVM). Support vector machines are supervised studying fashions with related mastering algorithms that analyze facts used for type and regression evaluation. Given a collection of education examples, each marked as belonging to 1 or the other of classes, an SVM schooling algorithm builds a variation that assigns new examples to 1 category or the substitute, making it a non-probabilistic binary mannequin. An SVM version is an illustration of the examples as factors in space, mapped so that the examples of the separate classes are divided by means of a clean hole that is as broad as practicable. New examples are then mapped into that equal discipline and expected to belong to a class-based absolutely on which side of the hole they fall.

In addition to acting linear class, SVMs can successfully perform a nonlinear type the use of what is called the kernel trick implicitly mapping their inputs into excessive-dimensional characteristic spaces. When information isn't classified, supervised learning isn't always feasible, and unmonitored gaining knowledge of approach is required, which tries to discover natural clustering of the statistics to companies, and then map new data to those formed companies. The aid vector clustering algorithm created by means of HavaSiegelmann and Vladimir Vapnik applies the information of assist vectors, advanced within the help vector machines algorithm, to categorize unlabeled facts, and is one of thewidely used clustering algorithms in industrial sector. The outcome of SVM returns the correct facial expression that has been classified from the given input image. 


\subsection{Algorithm}

Step 1: Get the Input Image.

Step 2: Pre-process the given input image using normalization and Gaussian blur function.

Step 3: Convert the given RGB image into Grey Scale Image. Step 4: Using Viola Jones Algorithm, first detect the facial region.

Step 5: Using the Viola Jones feature extractors, extract facial features separately.

Step 6: Invoke the SVM classifier by passing the facial features extracted in the above step.

Step 7: SVM classifier classifies the facial expression.

Step 8: Display the classified facial expression as output.

\section{EXPERIMENTAL RESULTS}

The experiment comprised of accepting an image or frame as an input. The face region is first extracted. This is followed by extracting the facial features and then these features are passed as inputs to the machine learning based classifier. In this experiment, SVM is used to classify the seven different facial expressions. The database used in this experiment is JAFFE which comprises of images containing different types of facial expressions. We selected 143 images comprising of all seven different facial expressions as training data. Another set of 150 images from the JAFFE database covering all the seven different facial expressions were used as test data.

The performance of the classifier was evaluated using the confusion matrix shown in Table 1 . The confusion matrix is a summary of result prediction on a classification problem. The number of incorrect and correct predictions are summarized with count values and broken down by each class. This is the key to the confusion matrix. The confusion matrix shows the different ways in which a classification model is confused when it makes predictions. It gives a clear picture not only into the errors being made by a classifier but also more importantly the types of errors that have occurred. In the below table, there are seven classes namely anger, disgust, worry, disappointment, happiness, wonder and neutral. Some important factors determine the efficiency of a confusion matrix are true positives, true negatives, false positives and false negatives.

True Positives (TP) denote a set of records correctly classified according to the correct class label. True Negatives
(TN) denote a set of negative records classified correctly under negative class bucket. The diagonal highlighted in bold in the above figure represents the true values while the remaining are false positives and negatives respectively. Table 1 shows the confusion matrix pertaining to the experiment conducted.

Based on these values, metrics such as Precision, Recall, Classification Rate and F-score can be derived. Precision (P) is denoted as follows:

$$
\text { Precision }=\frac{\mathrm{TP}}{\mathrm{TP}+\mathrm{FP}}
$$

Recall is given by:

$$
\text { Recall }=\frac{\mathrm{TP}}{\mathrm{TP}+\mathrm{FN}}
$$

The accuracy of a classifier is given by:

$$
\text { Accuracy }=\frac{T P+T N}{T P+T N+F P+F N}
$$

The average precision and recall values were 0.82 and 0.86 respectively. The overall accuracy of the classifier is $91 \%$. Figures 8 and 9 compares the precision and recall values with misclassification rate. It can be seen from the figures that the misclassification rate is very minimal and hence the classification model performs well in classifying facial expressions.

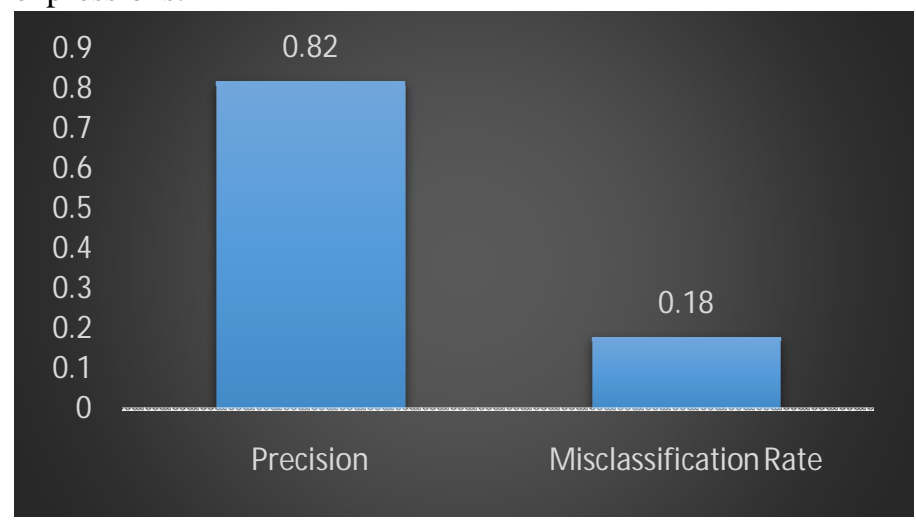

Figure 8: Precision vs Misclassification Rate

TABLE 1: CONFUSION MATRIX

\begin{tabular}{|l|c|c|c|c|c|c|c|c|}
\hline & ANGER & DISGUST & WORRY & DISAPPOINTM ENT & HAPPINESS & WONDER & NEUTRAL & TOTAL \\
\hline ANGER & 13 & 2 & 2 & 1 & 2 & 1 & 3 & 24 \\
\hline DISGUST & 2 & 8 & 1 & 1 & 3 & 1 & 2 & 18 \\
\hline WORRY & 3 & 1 & 14 & 4 & 2 & 1 & 1 & 26 \\
\hline DISAPPOINTMENT & 1 & 2 & 2 & 6 & 1 & 1 & 3 & 16 \\
\hline HAPPINESS & 2 & 1 & 1 & 1 & 12 & 4 & 3 & 24 \\
\hline WONDER & 1 & 1 & 1 & 2 & 3 & 9 & 2 & 19 \\
\hline NEUTRAL & 1 & 2 & 3 & 1 & 1 & 1 & 14 & 23 \\
\hline TOTAL & 23 & 17 & 24 & 16 & 24 & 18 & 28 & $\mathbf{1 5 0}$ \\
\hline
\end{tabular}




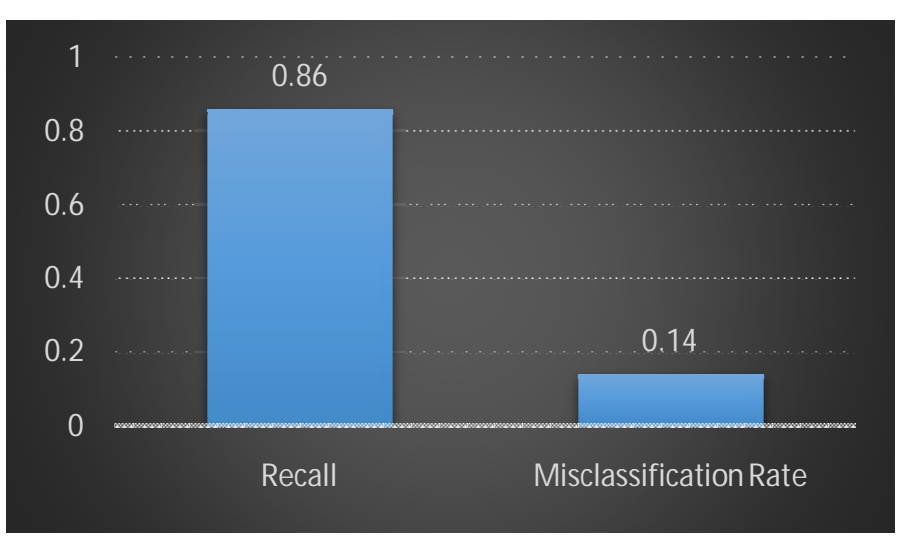

Figure 9: Recall vs Misclassification Rate

\section{CONCLUSION AND FUTURE WORK}

A classification model to classify the facial expressions into seven different categories was developed using Computer Vision and SVM. The proposed model was highly effective in classifying the facial expressions as observed by the high degree of accuracy in classification. Also, the precision and recall values of the proposed model is high while the misclassification rate is very low. This again is a good indicator of efficient classification of facial expressions.

Future work will be towards working on developing a model to detect composite facial expressions and to use this facial expression analysis for behavioral predictions.

\section{REFERENCES}

1. M. Pantic and I. Patras, "Dynamics of facial expression: recognition of facial actions and their temporal segments from face profile image sequences," in IEEE Transactions on Systems, Man, and Cybernetics, Part B (Cybernetics), vol. 36, no. 2, pp. 433-449, April 2006, doi: 10.1109/TSMCB.2005.859075.

2. Y. Li, S. Wang, Y. Zhao and Q. Ji, "Simultaneous Facial Feature Tracking and Facial Expression Recognition," in IEEE Transactions on Image Processing, vol. 22, no. 7, pp. 2559-2573, July 2013, doi: 10.1109/TIP.2013.2253477.

3. M. Dahmane and J. Meunier, "Prototype-Based Modeling for Facial Expression Analysis," in IEEE Transactions on Multimedia, vol. 16, no. 6, pp. 1574-1584, Oct. 2014, doi: 10.1109/TMM.2014.2321113.

4. M. Song, D. Tao, Z. Liu, X. Li and M. Zhou, "Image Ratio Features for Facial Expression Recognition Application," in IEEE Transactions on Systems, Man, and Cybernetics, Part B (Cybernetics), vol. 40, no. 3, pp. 779-788, June 2010, doi: 10.1109/TSMCB.2009.2029076.

5. M. Pantic and L. J. M. Rothkrantz, "Facial action recognition for facial expression analysis from static face images," in IEEE Transactions on Systems, Man, and Cybernetics, Part B (Cybernetics), vol. 34, no. 3, pp.1449-1461, June 2004, doi: 10.1109/TSMCB.2004.825931.

6. I. Kotsia and I. Pitas, "Facial Expression Recognition in Image Sequences Using Geometric Deformation Features and Support Vector Machines," in IEEE Transactions on Image Processing, vol. 16, no. 1, pp. 172-187, Jan. 2007, doi: 10.1109/TIP.2006.884954.

7. D. Li, Z. Li, R. Luo, J. Deng and S. Sun, "Multi-Pose Facial Expression Recognition Based on Generative Adversarial Network," in IEEE Access, vol. 7, pp. 143980-143989, 2019, doi: 10.1109/ACCESS.2019.2945423.

8. I. A. Essa and A. P. Pentland, "Coding, analysis, interpretation, and recognition of facial expressions," in IEEE Transactions on Pattern Analysis and Machine Intelligence, vol. 19, no. 7, pp. 757-763, July 1997, doi: 10.1109/34.598232.

9. Adena J. Zlochower, Jeffrey F. Cohn, James Jenn-Jier Lien, Takeo Kanade, Automated face coding: A computer-vision based method of facial expression analysis in parent-infant interaction, Infant Behavior and Development, Volume 21, Supplement, 1998, Page 16, ISSN 0163-6383.

10. MingliangXue, XiaodongDuan, Wanquan Liu, Yan Ren, A semantic facial expression intensity descriptor based on information granules, Information Sciences, Volume 528, 2020, Pages 113-132, ISSN 0020-0255.

11. Hamid Sadeghi, Abolghasem-A. Raie, Histogram distance metric learning for facial expression recognition, Journal of Visual Communication and Image Representation, Volume 62, 2019, Pages 152-165, ISSN 1047-3203.

12. Feng Zhou, Shu Kong, Charless C. Fowlkes, Tao Chen, Baiying Lei, Fine-grained facial expression analysis using dimensional emotion model, Neurocomputing, Volume 392, 2020, Pages 38-49, ISSN 0925-2312.

13. Manickam, M., Balasundaram, A., \&Ashokkumar, S. (2020). Structure optimized multi layer trespass perception system in cloud. International Journal on Emerging Technologies, 11(3), 77-81.

14. Magesh Kumar, S., Ashokkumar, S., \&Balasundaram, A. (2019). Providing enhanced resource management framework for cloud storage. International Journal of Engineering and Advanced Technology, 9(1), 3903-3908.

15. Subbiah S., Palaniappan S., Ashokkumar S., BalaSundaram A. (2020) A Novel Approach to View and Modify Data in Cloud Environment Using Attribute-Based Encryption. In: Ranganathan G., Chen J., Rocha Á. (eds) Inventive Communication and Computational Technologies. Lecture Notes in Networks and Systems, vol 89. Springer, Singapore. https://doi.org/10.1007/978-981-15-0146-3_20.

16. S. Magesh Kumar, Balasundaram A, Sathish Kumar P J. (2020). An Improved Optimization Algorithm for Word Search on Disk. International Journal of Control and Automation, 13(4), 952 - 957.

17. B. Ananthakrishnan, "An efficient approach for load balancing in cloud environment," International Journal of 
Scientific \& Engineering Research, vol. 6, no. 4, pp. 36-40, 2015.

18. Balasundaram, A., Chellappan, C. An intelligent video analytics model for abnormal event detection in online surveillance video. J Real-Time Image Proc 17, 915-930 (2020).

19. A. Balasundaram and C. Chellappan, "Vision Based Motion Tracking in Real Time Videos," 2017 IEEE International Conference on Computational Intelligence and Computing Research (ICCIC), Coimbatore, 2017, pp. 1-4, doi: 10.1109/ICCIC.2017.8524504.

20. A. Balasundaram, C. Chellappan, "Vision Based Gesture Recognition: A Comprehensive Study", The IIOAB Journal, Vol.8, Issue.4, pp.20-28, 2017.

21. Balasundaram, A., Ashok Kumar, S., \&Magesh Kumar, S. (2019). Optical flow based object movement tracking. International Journal of Engineering and Advanced Technology, 9(1), 3913-3916.

22. Balasundaram, A., \&Chellappan, C. (2019). Computer vision based system to detect abandoned objects. International Journal of Engineering and Advanced Technology, 9(1), 4000-4010.

23. Balasundaram, A. Computer Vision based Detection of Partially Occluded Faces. International Journal of Engineering and Advanced Technology, 9(3), 2188-2200.

24. Balasundaram, A, Ashokkumar, S. Study of Facial Expression Recognition using Machine Learning Techniques. JCR. 2020; 7(8): 2429-2437.

25. P.C.Senthil Mahesh et al., International Journal of Advanced Trends in Computer Science and Engineering, 9(3), May - June 2020, 3887 - 3891

26. PM Ashok Kumar et al., International Journal of Advanced Trends in Computer Science and Engineering, 9(3), May - June 2020, 3769 - 3775 